\title{
Heat transfer in solid halogenated methanes: trifluoromethane
}

\author{
V.A. Konstantinov, V.P. Revyakin, and V.V. Sagan \\ B. Verkin Institute for Low Temperature Physics and Engineering of the National Academy of Sciences of Ukraine \\ 47 Lenin Ave., Kharkov 61103, Ukraine \\ E-mail: konstantinov@ilt.kharkov.ua
}

Received January 4, 2009

\begin{abstract}
The isochoric thermal conductivity of solid trifluoromethane was investigated for three samples of different densities in the interval from $75 \mathrm{~K}$ to the onset of melting. The isochoric thermal conductivity first decreases with increasing temperature, passes through a minimum at $T \sim 100 \mathrm{~K}$, and then starts to increase slowly. The results obtained are compared with the thermal conductivities of other freons of methane series. The correlation between the temperature dependence of isochoric thermal conductivity and the character of the rotational molecular motion is discussed.
\end{abstract}

PACS: 66.70.-f Nonelectronic thermal conduction and heat-pulse propagation in solids; thermal waves; 63.20.Kk Phonon interactions with other quasiparticles.

Keywords: thermal conductivity, phonons, phonon-rotation coupling, diffusive modes.

\section{Introduction}

The solid freons of the methane series consisting of tetrahedral molecules are convenient objects to investigate the correlation between the rotational motion of molecules and the behavior of thermal conductivity. Methane $\left(\mathrm{CH}_{4}\right)$ and carbon tetrahalogenides $\left(\mathrm{CF}_{4}, \mathrm{CCl}_{4}, \mathrm{CBr}_{4}, \mathrm{CJ}_{4}\right)$ form high-temperature «plastic» or orientationally-disordered phases in which the rotational motion of molecules is similar to their motion in the liquid state [1,2]. In crystals consisting of lower-symmetry molecules such as chloroform $\left(\mathrm{CHCl}_{3}\right)$, methylene chloride $\left(\mathrm{CH}_{2} \mathrm{Cl}_{2}\right)$ or dichlorodifluoromethane $\left(\mathrm{CCl}_{2} \mathrm{~F}_{2}\right)$ the forces of the noncentral interaction of molecules are much stronger and the long-range order usually persists in them up to the melting temperature. The relative simplicity of the freon molecules allows adequate interpretation of experimental results.

The orientational motion in molecular crystals can be either vibrational or rotational depending on the temperature and the relationship between the noncentral forces and the kinetic energy of rotation. At low temperatures the motion of molecules in molecular crystals is, with rare exception (quantum crystals), of oscillatory character: at $T \rightarrow 0$ molecules perform zero orientational vibrations about the equilibrium directions. As the temperature rises, the r.m.s. amplitudes of librations increase and the molecules can hop over the accessible orientations. This disturbs the long-range order and can provoke a phase transition. The degree of orientational order can be varied by changing the temperature and selecting crystals with different parameters of the molecular interaction, which permits us to investigate the influence of the rotational motion of molecules upon the thermal properties of the crystal, including its thermal conductivity.

It is best to measure thermal conductivity at a fixed density, which excludes the effect of thermal expansion and ensures a more adequate comparison with theory. This is particularly important at high temperatures when the thermal expansion coefficients are large. Previously, we investigated the isochoric thermal conductivity of $\mathrm{CH}_{4}$ [3], $\mathrm{CCl}_{4}$ [4], $\mathrm{CHCl}_{3}$ and $\mathrm{CH}_{2} \mathrm{Cl}_{2}$ [5], $\mathrm{CF}_{2} \mathrm{Cl}_{2}$ and $\mathrm{CHF}_{2} \mathrm{Cl}$ [6]. The regularities of the heat transport in simple molecular crystals (including the methane-series freons) depending on the rotational degrees of freedom of molecules at the Debye and higher temperatures $\left(T \geq Q_{D}\right)$ can be generalized as follows. Because of the strong translational-orientational (TO) coupling in the orientationally-ordered phases [7], molecular librations contribute considerably to the thermal resistance $W=1 / \Lambda$ of the crystal. As a result, the isochoric thermal conductivity approaches its lower limit and deviates significantly from the dependence $\Lambda \propto 1 / T\left(\mathrm{CHCl}_{3}, \mathrm{CH}_{2} \mathrm{Cl}_{2}\right.$, and $\left.\mathrm{CF}_{2} \mathrm{Cl}_{2}\right)$. The concept of the lower limit of thermal conductivity 
implies that $\Lambda_{\min }$ can be achieved if the heat transport is realized through thermal energy diffusion between the neighboring quantum-mechanical oscillators whose lifetime is about half the oscillation period [8]. On a weakly-retarded rotation, the translational-orientational component of the total thermal resistance decreases owing to weakening of TO coupling and the isochoric thermal conductivity can grow with temperature. We observed this effect in methane and carbon tetrachloride $[3,4]$. A weak growth of thermal conductivity was also found in the high-temperature phase of solid chlorodifluoromethane $\left(\mathrm{CHF}_{2} \mathrm{Cl}\right)$ [6]. This behavior is not entirely clear for the lack of full information about the character of the rotational motion of the molecules in solid $\mathrm{CHF}_{2} \mathrm{Cl}$. According to calorimetric data [9], solid $\mathrm{CHF}_{2} \mathrm{Cl}$ experiences a $\lambda$-type phase transition at $59 \mathrm{~K}$ and melts at $115,7 \mathrm{~K}$ with an entropy jump $\Delta S_{f} / R=4,25$ ( $R$ is gas constant). Note that a crystal is defined as plastic if it obeys the Timmerman criterion: the melting entropy of phase I must be below 2,5 $R$. Calculation of the Debye temperature of $\mathrm{CHF}_{2} \mathrm{Cl}$ from calorimetric data gives $(70 \pm 5) \mathrm{K}$. The crystal structure of $\mathrm{CHF}_{2} \mathrm{Cl}$ was investigated at 10 and 70 $\mathrm{K}$ by the neutron scattering method [10]. The low-temperature phase is monoclinic; it has the spatial symmetry $P 112 / n\left(C_{2 h}^{4}\right)$ and eight molecules in the cell. The high-temperature phase is tetragonal with the spatial symmetry $\mathrm{P}_{2} / n\left(C_{2 h}^{4}\right)$ and it also has eight molecules in the cell. A shifting-type phase transition occurs at $59 \mathrm{~K}$ and has little effect on the position and orientation of the molecules. The object of this study was trifluoromethane $\left(\mathrm{CHF}_{3}\right)$. The molecule rotation at the lattice sites of $\mathrm{CHF}_{3}$ was investigated previously by the NMR technique $[11,12]$.

\section{Experimental}

Constant volume investigations are possible for molecular solids having a comparatively high compressibility coefficient. Using a high pressure cell, it is possible to grow a sample of sufficient density which in subsequent experiments can be cooled with practically unchanged volume. For samples of moderate densities the pressure drops during cooling to zero at a certain characteristic temperature $T_{0}$ and the isochoric condition is then broken. On further cooling, the sample can separate from the walls of the cell or its continuity can be disturbed. In the case of a fixed volume melting occurs in a certain temperature interval, and its onset shifts towards higher temperatures as the density of the samples increases.

The investigation was made using a steady-state technique in a coaxial-geometry setup [13]. The measuring beryllium bronze cell was $160 \mathrm{~mm}$ long with an inner diameter of $17.6 \mathrm{~mm}$. The maximum permissible pressure in it was $600 \mathrm{MPa}$. The inner measuring cylinder was $10.2 \mathrm{~mm}$ in diameter. The temperature sensors (platinum resistance thermometers) were placed in special channels of the inner and outer cylinders and thus escaped high-pressure effects. A system of protecting cylinders was used to reduce the axial heat flows. During the growth process the temperature gradient over the measuring cell was $2-3 \mathrm{~K} / \mathrm{cm}$. The pressure in the inflow capillary was varied within 50-150 MPa to grow samples of different densities. When the growth was completed, the capillary was blocked by freezing it with liquid hydrogen, and the samples were annealed for one to two hours at their premelting temperatures to remove the density gradients. After measurements the samples were evaporated into a thin-walled vessel and their masses were measure by weighing. The molar volumes of the samples were found from the known volume of the measuring cell and the sample masses. The total dominant systematic error of measurement was no more then $4 \%$ for the thermal conductivity and $0.2 \%$ for the volume. The purity of $\mathrm{CHF}_{3}$ was no worse than $99.98 \%$.

\section{Results and discussion}

The isochoric thermal conductivity of solid trifluoromethane was investigated on three samples of different densities in the interval from $75 \mathrm{~K}$ to the onset of melting. The experimental results are shown in Fig. 1 (solid lines are smoothed thermal conductivity values; the dashed line shows the thermal conductivity measured under saturated vapor pressure). The molar volumes $V_{m}$, temperatures $T_{0}$ (onset of $V=$ const condition) and $T_{m}$ (onset of sample melting) are shown in Table 1. The Bridgman coefficients $g=-(\partial \ln \Lambda / \partial \ln V)_{T}$ calculated from the experimental results are $4.8 \pm 0.8$ at $T=115 \mathrm{~K}$. Isochoric thermal

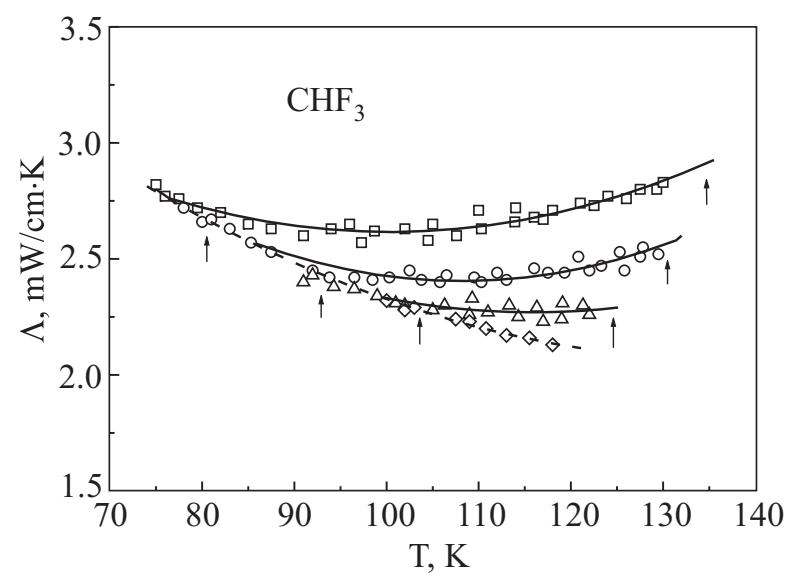

Fig. 1. The isochoric thermal conductivity of three solid $\mathrm{CHF}_{3}$ samples of different densities: Nos. $1(\Delta), 2(\mathrm{O}), 3(\square)$ (see also Table 1). Solid lines show smoothed values of isochoric thermal conductivity. Dashed line and rhombus are for the thermal conductivity of a free sample. Arrows mark the onset of the experimental condition $V=$ const and the onset of the sample melting. 
Table 1. Molar volumes $V_{m}$ of the samples, temperatures $T_{0}$ of the onset of experimental condition $V=$ const and temperatures $T_{m}$ of the onset of melting

\begin{tabular}{c|c|c|c}
\hline \hline Number of sample & $V_{m}, \mathrm{~cm}^{3} / \mathrm{mole}$ & $T_{0}, \mathrm{~K}$ & $T_{m}, \mathrm{~K}$ \\
\hline \hline 1 & 36.58 & 104 & 125 \\
\hline 2 & 35.9 & 93 & 131 \\
\hline 3 & 35.45 & 82 & 135 \\
\hline \hline
\end{tabular}

conductivity first decreases with increasing temperature, passes through a minimum at $T \sim 100 \mathrm{~K}$, and then starts to increase slowly up to the onset of melting.

Trifluoromethane $\mathrm{CHF}_{3}$ melts at $T_{m}=117.97 \mathrm{~K}$, the melting entropy being $\Delta S_{f} / R=4.14$ [14]. According to heat capacity data [14], there are no phase transitions in the interval $15-117 \mathrm{~K}$. We estimated the Debye temperature $\left(\Theta_{D}=88 \pm 5 \mathrm{~K}\right)$ from the low-temperature part of these data. The existence of only one crystalline modification in the region $20-106 \mathrm{~K}$ is also supported by Raman and IR spectral investigations [15]. This points to the absence of strong hydrogen bonds in $\mathrm{CHF}_{3}$. Neutron scattering investigations of the crystallographic structure of $\mathrm{CHF}_{3}$ at $4.2,40$ and $70 \mathrm{~K}$ [16] revealed only one crystalline phase of the spatial symmetry $P 2{ }_{1} / c$ with four differently oriented molecules in the monoclinic cell.

Absorption line shapes of the NMR signal and the spin-lattice relaxation times were investigated on the ${ }^{1} \mathrm{H}$ and ${ }^{19} \mathrm{~F}$ nuclei of $\mathrm{CHF}_{3}$ at $T=7-116 \mathrm{~K}$ [11] and $4.2-120$ $\mathrm{K}$ [12], respectively. It is found that above $T=80 \mathrm{~K}$ the second NMR moment decreases sharply from $11.5 \mathrm{G}^{2}$ to $3.0 \mathrm{G}^{2}$ immediately prior to melting, which suggests enhancement of the molecule rotation about the three fold axis. The activation energy of rotation and the preexponential Arrhenius factors were calculated to be $17.0 \mathrm{~kJ} / \mathrm{mol}$ and $1.0 \cdot 10^{-16} \mathrm{~s}[11]$, and $18.0 \mathrm{~kJ} / \mathrm{mol}$ and $(4.9-8.9) \cdot 10^{-17} \mathrm{~s}$ [12], respectively. In Fig. 2 these results are presented as a logarithmic function of frequency of reorientations about the three fold axis versus the inverse temperature. The upper frequency of the lattice modes which correspond to $\Theta_{D}=88 \mathrm{~K}$ is shown at the top of Fig. 2. The data of the two studies are in good agreement. At the melting temperature the reorientation frequency is three orders of magnitude lower than the Debye frequency, which is rather surprising. The growth of thermal conductivity with temperature was observed previously in orientationally-disordered phases of methane and carbon tetrahalogenides, in which the molecule rotation is weakly hindered or almost free $[1,2]$.

Table 2 gives a general information about the methane-series freons: melting temperatures $T_{m}$, phase transition temperatures $T_{\text {I-II }}$, structure and the number of molecules in the unit cell $z$, melting entropy $\Delta S_{f} / R$, Debye temperatures $\Theta_{D}$, Bridgman coefficients $g=-(\partial \ln \Lambda / \partial \ln V)_{T}$, dipole

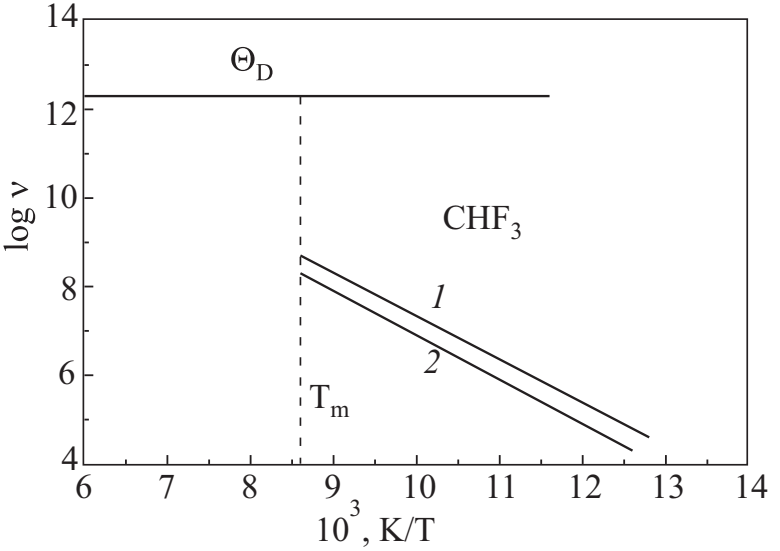

Fig. 2. Frequency of reorientation jumps of molecules in crystalline $\mathrm{CHF}_{3}$ for to studies according to Refs. 11, 12 .

moments of molecules $\mu$. Included are also the data for $\mathrm{CBr}_{4}$ (its thermal conductivity was measured in Ref. 17). The isochoric thermal conductivity of orientationally-disordered phases in the methane-series freons increases with temperature. (The exception is methane in where the

Table 2. Melting temperatures $T_{m}$, phase transition temperatures $T_{\text {I-II }}$, structure and the number of molecules per unit cell $z$, melting entropy $\Delta S_{f} / R$, Debye temperatures $\Theta_{D}$ (the corresponding temperature is given in brackets), Bridgman coefficients $g$, dipole moments of molecules $\mu$

\begin{tabular}{|c|c|c|c|c|c|c|c|}
\hline Substance & $T_{m}, T_{\mathrm{I}-\mathrm{II}}$ & Structure & $z$ & $\Delta S_{f} / R$ & $\Theta_{D}, \mathrm{~K}$ & $g$ & $\mu, D$ \\
\hline (I) & 90.6 & $F_{m} 3_{m}$ & 4 & & $96(90 \mathrm{~K})$ & 8.8 & \\
\hline $\mathrm{CH}_{4}$ & & - & & 1.24 & & & 0 \\
\hline (II) & 20.5 & $P 43 m$ & 32 & & $141(0 \mathrm{~K})$ & & \\
\hline (I) & 250.3 & $F_{m} 3_{m}$ & 4 & & & 6.0 & \\
\hline $\mathrm{CCl}_{4}$ & & & & 1.21 & & & 0 \\
\hline (II) & 225.5 & $C 2 / c$ & 32 & & $92(0 \mathrm{~K})$ & 6.5 & \\
\hline (I) & 363 & $F_{m}{ }^{3} m$ & 4 & & & 3.8 & \\
\hline $\mathrm{CBr}_{4}$ & & & & 1.3 & $62(300 \mathrm{~K})$ & & 0 \\
\hline (II) & 320 & $C 2 / c$ & 32 & & & 3.4 & \\
\hline (I) & 115.7 & $P 4_{2} / n$ & 8 & & & 4.5 & \\
\hline $\mathrm{CHF}_{2} \mathrm{Cl}$ & & & & 4.25 & & & 1.41 \\
\hline (II) & 59 & $P 112 / n$ & 8 & & $70(0 \mathrm{~K})$ & & \\
\hline $\mathrm{CHCl}_{3}$ & 210 & Pnma & 4 & 5.4 & $86^{*}$ & 3.9 & 1.01 \\
\hline $\mathrm{CH}_{2} \mathrm{Cl}_{2}$ & 176 & Pbcn & 4 & 3.13 & $115^{*}$ & 4.6 & 1.6 \\
\hline $\mathrm{CF}_{2} \mathrm{Cl}_{2}$ & 115 & $F d d 2$ & 8 & 4.2 & $80^{*}$ & 5.0 & 0.51 \\
\hline $\mathrm{CHF}_{3}$ & 118 & $P 2_{1} / c$ & 4 & 4.14 & $88^{*}$ & 4.6 & 1.6 \\
\hline
\end{tabular}

*Estimates obtained from IR and Raman spectra for the upper boundary of the lattice modes. 
thermal conductivity has a maximum and then starts to decrease [3].) This is because the translational-orientational component of the total thermal resistance decreases sharply as the rotation of the molecules becomes weakly hindered. In some crystals with reorientation frequencies up to $10^{4} \mathrm{~s}^{-1}\left(\mathrm{CHCl}_{3}, \mathrm{CH}_{2} \mathrm{Cl}_{2}\right.$ and $\left.\mathrm{CF}_{2} \mathrm{Cl}_{2}\right)$ the isochoric thermal conductivity approaches its lower limit $\Lambda_{\min }[5,6]$ and deviates significantly from the dependence $\Lambda \propto 1 / T$.

Apart from $\mathrm{CHF}_{3}$, a weak growth of isochoric thermal conductivity with temperature was also observed in $\mathrm{CHF}_{2} \mathrm{Cl}$ (I) [6]. However, in the latter case only the Raman spectra were investigated at $T=20-80 \mathrm{~K}$ [18] and NMR data are unavailable. The lattice modes exhibit distinct peaks at $T=20 \mathrm{~K}$ and broad overlapping bands at $T=80 \mathrm{~K}$, which are more common for orientationally-disordered crystals. At $20 \mathrm{~K}$ there were three split peaks in the interval $30-50 \mathrm{~cm}^{-1}(43-72 \mathrm{~K})$ and three split peaks in the region $55-80 \mathrm{c}^{-1}(79-115 \mathrm{~K})$. They were presumably assigned to the translational and librational modes, respectively. The upper boundary of the translational modes agrees well with the Debye frequency obtained from calorimetric data. However, the analysis of the spectra of the intramolecular modes at $80 \mathrm{~K}$ reveals no disordered structure. The observed picture might therefore be a result of broadening and re-overlapping of the corresponding components. We can expect intensive reorientational motion in solid $\mathrm{CHF}_{2} \mathrm{Cl}$ at least about the $\mathrm{C}-\mathrm{Cl}$ axis.

The weak growth of isochoric thermal conductivity with temperature in solid $\mathrm{CHF}_{3}$ and $\mathrm{CHF}_{2} \mathrm{Cl}$ suggests that the translational-orientational coupling becomes weaker in these crystals at premelting temperatures owing to the intensive molecule reorientations about the three fold axes. It is rather surprising that in $\mathrm{CHF}_{3}$ the reorientation frequency is much lower than the boundary Debye one. This fact calls for further theoretical studies. At present there is no general theory that could relate the behavior of thermal conductivity to the character of the orientational motion of molecules in molecular crystals.
1. N.G. Parsonage and L.A.K. Staveley, Disorder in Crystals, Clarendon Press, Oxford (1978).

2. The Plastically Crystalline State. Orientationally-Disordered Crystals, Y.N. Sherwood (ed.), John Wiley \& Sons, Chichester-New York-Brisbane-Toronto (1979).

3. V.A. Konstantinov, V.G. Manzhelii, V.P. Revyakin, and S.A. Smirnov, Physica B262, 421 (1999).

4. V.A. Konstantinov, V.G. Manzhelii, and S.A. Smirnov, Phys. Status Solidi B163, 369 (1991).

5. V.A. Konstantinov, V.G. Manzhelii, and S.A. Smirnov, Fiz. Nizk. Temp. 17, 883 (1991) [Low Temp. Phys. 17, 462 (1991)].

6. V.A. Konstantinov, V.G. Manzhelii, S.A. Smirnov, and V.P. Revyakin, Fiz. Nizk. Temp. 21, 102 (1995) [Low Temp. Phys. 21, 78 (1995)].

7. R.M. Lynden-Bell and K.H. Michel, Rev. Mod. Phys. 66, 721 (1994).

8. D.G. Cahill, S.K. Watson, and R.O. Pohl, Phys. Rev. B46, 6131 (1992).

9. E.F. Neilson and D. White, J. Am. Chem. Soc. 79, 5618 (1957).

10. O.S. Binbrek, B.H. Torrie, R. Dreele, and B.M. Powell, Molec. Phys. 90, 49 (1997).

11. T. Eguchi, M. Kishita, H. Chihara, and G. Soda, Bull. Chem. Soc. Jpn. 55, 676 (1982).

12. A. Watton, J.C. Pratt, E.C. Reynhardt, and H.E. Petch, J. Chem. Phys. 77, 2344 (1982).

13. V.A. Konstantinov, S.A. Smirnov, and V.P. Revyakin, Instr. Exp. Tech. 42, 133 (1999).

14. R.H. Valentine, G.E. Brodale, and W.F. Giauque, J. Phys. Chem. 66, 392 (1962).

15. B Andrews, A. Anderson, and B.H. Torrie, Chem. Phys. Lett. 105, 551 (1984).

16. B.H. Torrie, O.S. Binbrek, and B.M. Powell, Mol. Phys. 87, 1007 (1996).

17. P. Andersson and R.G. Ross, Mol. Phys. 39, 1359, (1980).

18. J.H. Lefebvre and A. Anderson, J. Raman Spectr. 23, 243 (1992). 